\title{
Predictions on the Number of Variable Stars for the GAIA Space Mission and for Surveys such as the Ground-Based International Liquid Mirror Telescope
}

\author{
Laurent Eyer ${ }^{1}$, Jan C'uypers ${ }^{2}$
}

\begin{abstract}
Future space and ground-based survey programmes will produce an impressive amount of photometric data. The GAIA space mission will map the complete sky down to $V=20^{\mathrm{m}}$, and produce time series for about 1 billion stars. Survey instruments such as the International Liquid Mirror Telescope will observe slices of the sky down to $V=23^{\mathrm{m}}$. In both experiments, the opportunity exists to discover a huge number of variable stars. Predictions of the expected total number of variable stars and the number of variables in specific subgroups are given.
\end{abstract}

\section{The Total Number of Variable Stars}

A first estimate of the total number of variable stars observable by GAIA was done by Eyer (1999). The star population used came from the star-count model of Torra et al. (1999) and the variability detection threshold was derived from the Hipparcos survey results. With the new specifications of the GAIA mission, about $10^{9}$ stars (up to mag $G<20$ ) are expected to be observed, with about 18 million variable stars, including about 5 million "classic" periodic variables. Very different star counts are obtained according to the extinction laws used (Figueras, private communication). Since the quality of the GAIA photometry in the crowded fields is still uncertain, we cannot discuss here the number of variables in dense clusters and galaxies. About 2-3 million eclipsing binaries will be observed (their detection probability will be studied in detail in the future), and $\sim 3 \times 10^{5}$ stars with rotation-induced variability can be expected.

\section{The Methods}

For a specific interval of $V-I$, we computed the proportion of variables in the Hipparcos survey and we applied that rate to the number of stars obtained from the Figueras model (method A). Surface densities were calculated, either from the Hipparcos parallaxes or from the specific properties of the stars. We integrated and removed the stars behind the bulge (method B). We extrapolated the GCVS data (Kholopov et al. 1998), assuming detection completeness up to a certain magnitude and a magnitude limit for the population beyond which no

\footnotetext{
${ }^{1}$ Instituut voor Sterrenkunde, K. U. Leuven, Celestijnenlaan 200B, B-3001 Heverlee, Belgium

${ }^{2}$ Royal Observatory of Belgium, Ringlaan 3, B-1180 Brussel, Belgium
} 
more stars are present (method $\mathrm{C}$ ). We also analysed the detection rates of the microlensing surveys (when available) and scanned the literature.

\section{Pulsating Variables}

Methods $A$ and $C$ estimate the number of $\beta$ Cephei stars to be about 3000 . 15000 SPB variables will be detected according to method A. Applying methods $A$ and $C$ gave about the same estimate for $\delta$ Scuti stars: 60000 . However, it will be very difficult to analyse the very reddened, low-amplitude variables. With method B even higher numbers (240000) of $\delta$ Scuti stars show up. With a total number of RR Lyrae as given by Suntzeff, Kinman, \& Kraft (1991) we arrive at 70000 observable RR Lyrae stars (method B). Using the OGLE and MACHO detection rates, we expect 15000 to $40000 \mathrm{RR}$ Lyrae stars in the bulge.

All galactic Cepheids are within the observational range of GAIA, if they are not too obscured by interstellar extinction. Results of recent deep surveys confirm the early estimates of a total of 2000 to 8000 Cepheids. With the help of the database (Fernie et al. 1995), we obtained (method B) a density of 15-20 Cepheids $/ \mathrm{kpc}^{2}$, leading to an estimate of 5 200-6900 observable stars. Early estimates gave a total of 200000 Miras and related long period variables in the Galaxy. With 500 Miras $/ \mathrm{kpc}^{2}, 140000$ to 170000 Miras will be observable. Method B gave us a density of $250-350$ semiregular variables $/ \mathrm{kpc}^{2}$ for a total of 100000 observable SR stars.

We plan to calculate and analyse all categories of variable stars in more detail to arrive at reliable estimates of all observable variable stars in the Galaxy.

\section{Variable Stars in Deep Surveys: The International Liquid Mirror Telescope (ILMT)}

An international group of institutions is actively interested in developing a 4-m class liquid mirror telescope. If the view of the ILMT includes fields near the galactic centre, and if all stars from $17 \leq R \leq 20$ mag can be measured with high precision $(\sigma \leq 0.01 \mathrm{mag})$, the project will yield time series for about 2 million stars with 500 measurements per star during $5 \mathrm{yr}$. About 10000 new variable stars can be expected, including 6000 faint eclipsing binaries, $200 \mathrm{RR}$ Lyrae stars and 300 long period variables.

(see http://vela.astro.ulg.ac.be/themes/telins/lmt/index_e.html)

\section{References}

Eyer, L. 1999, Balt. Ast., 8, 321

Fernie, J. D., Beattie, B., Evans, N. R., \& Seager, S. 1995, IBVS, No. 4148

GAIA: http://astro.estec.esa.nl/SA-general/Projects/GAIA/

HIPPARCOS: Hipparcos and Tycho catalogues, ESA SP-1200

Kholopov, P. N., Samus, N. N., et al. 1998, The Combined General Catalogue of Variable Stars, 4.1 Edition, Moscow

Suntzeff, N. B., Kinman, T. D., \& Kraft, R. P. 1991, ApJ, 367, 528

Torra, J., Chen, B., Figueras, F., Jordi, C., \& Luri, X. 1999, Balt. Astr., 8, 171 